\title{
Como a pandemia de Covid-19 afetou o tratamento odontopediátrico nos serviços públicos de saúde? Análise de dados do Estado de Goiás
}

\author{
Maria Millena Carneiro FLORÊNCIO'; Gabriella Garcia VAZ1; \\ Robertha Lyssa Ferreira ARAÚJO'; Patrícia CORRÊA-FARIA² \\ 1 - Graduanda em Odontologia, Faculdade Sul-Americana, Goiânia-GO, Brasil. \\ 2 - Professora do Departamento de Odontologia, Faculdade Sul-Americana, Goiânia-GO, Brasil.
}

\section{Resumo}

Objetivos: Avaliar o impacto da pandemia de COVID-19 no tratamento odontológico de crianças atendidas no serviço público em Goiás e a relação entre o número de casos da doença e de procedimentos odontopediátricos. Material e método: Dados sobre o número de procedimentos odontológicos e de casos confirmados de COVID-19 foram obtidos em consulta aos relatórios disponibilizados pelo Ministério da Saúde e boletins epidemiológicos de órgãos sanitários. A diferença no número de procedimentos foi calculada: 1) comparação do número de procedimentos realizados em fevereiro e março/2020, quando foi confirmado o primeiro caso em Goiás; 2) e do número de procedimentos realizados em cada mês de $2020 \mathrm{com}$ seu correspondente em 2019. Os resultados foram obtidos em número absoluto e em porcentagem de aumento/redução. Avaliou-se a correlação entre o número de procedimentos e de casos de COVID-19. Resultados: No primeiro mês da pandemia, houve redução de $24 \%$ no total de procedimentos. Maior redução foi observada para as restaurações (28\%) e extrações (21\%). Ao comparar os meses de 2020 com 2019 , houve redução de até $81 \%$ nos procedimentos; no segundo semestre a redução variou entre $76 \%$ e $83 \%$. Houve redução de até $100 \%$ do número de restaurações. Menor redução foi observada para os procedimentos endodônticos (23 a 88\%). Houve correlação negativa entre o número de procedimentos e de casos de COVID-19 (rho -0,806; $p=0,005$ ). Conclusões: A pandemia impactou negativamente o número de procedimentos odontopediátricos realizados no SUS, em Goiás. Maior número de casos de COVID-19 se relacionou ao menor número de procedimentos odontopediátricos.

PALAVRAS-CHAVE: Infecções por coronavírus; Assistência odontológica; Sistema Único de Saúde. 


\section{Introdução}

Aproximadamente cinco em cada dez crianças têm cárie em dentes decíduos ${ }^{1}$. Dentre essas, $9 \%$ têm lesões não tratadas ${ }^{2}$ e experimentam dor, impacto negativo no crescimento e desenvolvimento, e na qualidade de vida relacionada à saúde bu$\mathrm{cal}^{3,4}$. Outras condições bucais como traumatismo dentário e maloclusões também são frequentes. Cerca de duas em cada dez crianças têm traumatismo dentário ${ }^{5}$; e, pelo menos metade tem maloclusão ${ }^{6}$.

Consequências sociais e econômicas são observadas como um resultado dos problemas bucais em crianças $^{7,8}$. Estresse, faltas ao trabalho, gastos com transporte e com consultas odontológicas, e uso inapropriado de medicamentos de venda livre para alívio da dor são morbidades associadas à família ${ }^{7}$. O custo do tratamento odontológico, realizado algumas vezes em nível hospitalar e sob anestesia geral; e de medicamentos como analgésicos e antibióticos são fatores importantes na avaliação do impacto das condições bucais nos sistemas de saúde ${ }^{7}$.

O tratamento odontológico é uma das estratégias para reduzir os impactos negativos ${ }^{9}$ e auxiliar na interrupção do processo de doença, quando somado às medidas preventivas, ao diagnóstico e intervenções precoces ${ }^{10}$. No Brasil, atendimento odontológico gratuito é oferecido à população por meio do Sistema Único de Saúde, desde 2004, a partir da iniciativa Brasil Sorridente ${ }^{11}$. Apesar de o serviço estar disponível, as visitas odontológicas por crianças ainda são subutilizadas ${ }^{12}$ e pouca prioridade ou atenção é dada àquelas em idade pré-escolar ${ }^{13}$. Quando há procura por tratamento, a principal queixa é a presença de cárie ${ }^{14,15}$.

A baixa procura por tratamento odontológico de crianças se deve as barreiras relacionadas a fatores econômicos, culturais e à organização dos serviços ${ }^{13}$. Desde março de 2020, a pandemia de COVID-19 se somou a estes fatores. A transmissão da COVID-19 se dá por meio de gotículas respiratórias ou por contato das mãos contaminadas com a mucosa da boca, 
do nariz ou dos olhos ${ }^{16}$. Os dentistas se tornaram profissionais com grande risco de contaminação, uma vez que manipulam a cavidade bucal do paciente, em contato direto com saliva, e realizam procedimentos geradores de aerossóis ${ }^{17}$. Para minimizar o risco de contaminação, houve uma considerável redução no número de atendimentos odontológicos e adiamento dos procedimentos eletivos ${ }^{18}$. Esta medida resultou na redução do número de procedimentos odontopediátricos realizados nos serviços públicos de saúde, em todo o Brasil ${ }^{19}$.

Um estudo recentemente publicado revelou que, em abril de 2020 (período de contágio exponencial), houve uma redução de $89 \%$ no número total de procedimentos ${ }^{19} \mathrm{e}$, possivelmente, agravamento da condição de saúde bucal das crianças atendidas no serviço público de saúde. Neste estudo foram avaliados dados secundários correspondentes ao período de janeiro/2019 a maio/2020 e os resultados foram estratificados para as cinco regiões do Brasil. A região centro-oeste foi a terceira com maior redução no número de procedimentos. As regiões Sul e Sudeste foram as outras com maior redução do número de procedimentos odontopediátricos realizados nos serviços públicos de saúde ${ }^{19}$.

Até o momento, parece não haver investigações sobre a redução dos procedimentos no estado de Goiás. Os dados disponíveis se referem à região centro-oeste como um todo ${ }^{19}$. Informações sobre o impacto da pandemia de COVID-19 no tratamento odontopediátrico podem orientar a formulação de estratégias para a retomada e priorização dos atendimentos. Estas medidas devem ser elaboradas considerando as particularidades de cada região. Diante disso, os objetivos deste estudo são verificar o impacto da pandemia de COVID-19 nos atendimentos odontopediátricos realizados no sistema público de saúde, no estado de Goiás e investigar a relação entre o número de casos da doença e de procedimentos. 


\section{Material e método}

Trata-se de um estudo observacional longitudinal retrospectivo ecológico realizado a partir de dados secundários obtidos dos relatórios públicos do Departamento de Informática do Sistema Único de Saúde (DATASUS), do Ministério da Saúde do Brasil, e de boletins epidemiológicos publicados periodicamente pela Superintendência de Vigilância em Saúde e Gerência de Vigilância Epidemiológica do estado de Goiás ${ }^{20}$.

Foram extraídas da base eletrônica de dados, as informações sobre o número mensal de procedimentos - restaurações, tratamentos endodônticos e extrações - realizados em dentes decíduos no período de janeiro de 2019 a dezembro de 2020 nas unidades de saúde do Sistema Único de Saúde (SUS), em Goiás. Os boletins epidemiológicos foram consultados para se obter o número de casos confirmados de COVID-19 em cada mês de avaliação.

Os dados foram inseridos e analisados no software Statistical Package for Social Sciences (SPSS for Windows, versão 25.0, SPSS Inc., Chicago, IL, USA). A diferença no número de procedimentos foi calculada de duas formas: 1) a partir da comparação do número de procedimentos realizados em fevereiro (mês anterior à pandemia) e março de 2020 (quando foi confirmado o primeiro caso de infecção pelo coronavírus em Goiás); 2) comparação do número de procedimentos realizados em cada mês do ano 2020 com seu correspondente em 2019. A diferença no número de procedimentos foi calculada por meio de número absoluto (resultado da subtração) e em porcentagem de aumento ou de redução. A análise foi realizada para cada tipo de procedimento e considerando-se cada procedimento isoladamente.

A relação linear entre o número de casos de COVID-19 e de procedimentos realizados no período de março a dezembro de 2021 foi testada por meio do teste de correlação de Spearman. Um nível de significância de 5\% foi adotado. 


\section{Resultados}

Uma redução de $24,0 \%$ no número de procedimentos realizados em dentes decíduos foi observada ao se comparar os meses de fevereiro e março/2020. Maiores porcentagens de redução foram observadas nas comparações realizadas entre cada mês do ano de 2020 e seu correspondente em 2019. Em 2020, houve uma redução de até $81 \%$ no número de procedimentos realizados em dentes decíduos; para procedimentos restauradores houve redução de até $100 \%$. O número de exodontias sofreu redução de até $75 \%$. Menor porcentagem de redução foi observada nos procedimentos endodônticos, em todos os meses desde a confirmação do primeiro caso de COVID-19 no estado (Tabela 1). Números absolutos de procedimentos realizados nos períodos anteriores e posteriores à confirmação do primeiro caso da doença são apresentados na Figura 1.

FIGURA 1 - Número absoluto de procedimentos odontológicos realizados mensalmente em 2019 e em 2020

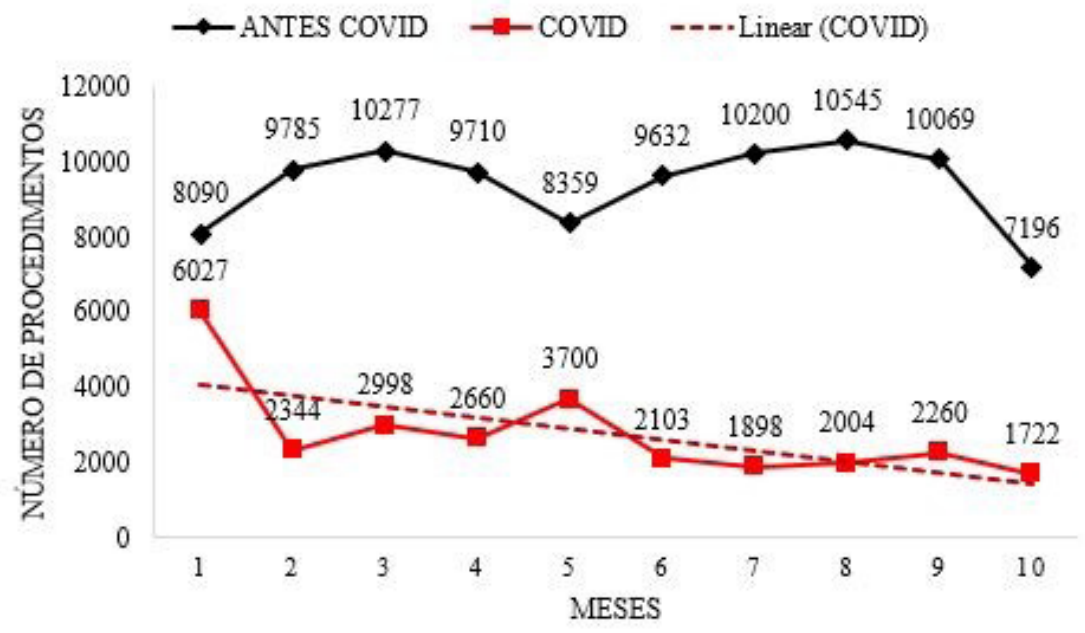

1 a 10 indicam os meses de março a dezembro/2020. Os valores apresentados na parte superior do gráfico se referem ao ano de 2019 e aqueles localizados na parte inferior, ao ano de 2020.

A correlação entre o número total de procedimentos realizados e o número de casos confirmados de COVID-19 foi negativa, forte e significativa (rho $-0,806 ; \mathrm{p}=0,005)$. 
TABELA 1 • Porcentagem de redução/aumento do número de procedimentos odontológicos realizados no período de 2019/2020 e número de casos confirmados de COVID-19.

\begin{tabular}{|c|c|c|c|c|c|}
\hline MESES & $\begin{array}{l}\text { Procedimentos } \\
\text { endodônticos }\end{array}$ & Restaurações & Exodontias & $\begin{array}{l}\text { Total de } \\
\text { procedimentos }\end{array}$ & $\begin{array}{l}\text { Número } \\
\text { de casos } \\
\text { confirmados de } \\
\text { COVID-19* }\end{array}$ \\
\hline Março/2020 & $+51 \%$ & $-31 \%$ & $-26 \%$ & $-26 \%$ & 65 \\
\hline Abril/2020 & $+16 \%$ & $-67 \%$ & $-68 \%$ & $-76 \%$ & 661 \\
\hline Maio/2020 & $-43 \%$ & $-82 \%$ & $-57 \%$ & $-71 \%$ & 2671 \\
\hline Junho/2020 & $-30 \%$ & $-77 \%$ & $-75 \%$ & $-73 \%$ & 17476 \\
\hline Julho/2020 & $-21 \%$ & $-66 \%$ & $-49 \%$ & $-56 \%$ & 55796 \\
\hline Agosto/2020 & $-30 \%$ & $-100 \%$ & $-54 \%$ & $-78 \%$ & 116387 \\
\hline Setembro/2020 & $-30 \%$ & $-97 \%$ & $-65 \%$ & $-81 \%$ & 184977 \\
\hline Outubro/2020 & $-30 \%$ & $-97 \%$ & $-64 \%$ & $-81 \%$ & 247386 \\
\hline Novembro/2020 & $-23 \%$ & $-94 \%$ & $-57 \%$ & $-78 \%$ & 273456 \\
\hline Dezembro/2020 & $-25 \%$ & $-91 \%$ & $-61 \%$ & $-76 \%$ & 309317 \\
\hline
\end{tabular}

*Número de casos confirmados registrado no último boletim epidemiológico publicado em cada mês do ano de 2020

\section{Discussão}

Este estudo observacional ecológico teve como objetivo verificar o impacto da pandemia de COVID-19 no número de procedimentos odontopediátricos realizados no serviço público de saúde em Goiás. Comparações entre o número de procedimentos realizados em fevereiro/2020, mês anterior à pandemia, e março foram realizadas; adicionalmente, comparou-se o número de procedimentos realizados, a cada mês, no ano de 2020 e no período correspondente, em 2019 . Houve redução de até $81 \%$ no número total de procedimentos, sendo que as restaurações foram os procedimentos que sofreram maior redução. A redução do número de procedimentos se relacionou ao aumento dos casos de COVID-19.

Os resultados sobre a redução do número de procedimentos estão de acordo com estudo anterior em que foram avaliados dados secundários de cada uma das regiões do Brasil. No estudo 
citado, houve redução de $66 \%$ na época correspondente ao primeiro caso de COVID-19 e de 89\% na época de contágio exponencial ${ }^{19}$. Em Goiás, a porcentagem de redução de 81\%, em 2020, se aproximou ao observado no estudo anterior. $\mathrm{Na}$ época correspondente ao primeiro caso de COVID-19, a porcentagem de redução foi menor. A comparação entre os achados dos estudos deve ser realizada com cautela, uma vez que, na investigação anterior, não é possível isolar os resultados correspondentes ao estado de Goiás.

A suspensão das atividades odontológicas na rede pública foi solicitada pelo Conselho Federal de Odontologia (CFO) ao Ministério da Saúde (MS), em março de 202021. o mesmo movimento foi observado em países como Estados Unidos, Escócia e Irlanda do Norte ${ }^{22}$. Desde então, apenas atendimentos de urgência e emergência permaneceram autorizados, desde que as medidas relativas aos processos de desinfecção, esterilização e uso de equipamentos de proteção individual fossem rigorosamente seguidas. Isso parece justificar a redução imediata do número de procedimentos odontológicos, especialmente das restaurações que, a depender da situação clínica podem ser consideradas procedimentos eletivos. É possível que apenas as crianças com dor ${ }^{23,24}$ tenham sido tratadas neste período da pandemia.

Até dezembro/2020, os procedimentos endodônticos foram os que tiveram menor porcentagem de redução. Esses procedimentos são indicados em dentes decíduos principalmente em casos de extensas lesões cariosas com envolvimento do tecido pulpar, abcessos, infecções dentárias e em alguns casos de traumatismo. Estas queixas são consideradas urgentes e devem ser prontamente atendidas, mesmo durante a pandemia ${ }^{25}$. Tal fato, justifica a menor porcentagem de redução em procedimentos endodônticos.

O número absoluto de procedimentos endodônticos se manteve constante durante o período avaliado neste estudo. Este achado sugere um possível agravamento da condição bucal das crianças. 
Pacientes com lesões de cárie que poderiam ser tratadas com procedimentos minimamente invasivos e/ou restauradores podem ter tido piora na condição bucal, necessitando de procedimentos mais invasivos como a pulpectomia e a extração.

A progressão das lesões de cárie, durante a pandemia, pode ser evitada por meio do controle dos fatores de risco ${ }^{26}$, do uso de fluoretos e procedimentos capazes de paralisá-las, como a aplicação de substâncias cariostáticas ${ }^{27}$. Quando estas medidas não são adotadas de forma eficiente, o agravamento das lesões causa dor e impacta negativamente na qualidade de vida da criança ${ }^{4} \mathrm{e}$ no sistema de saúde, gerando maiores gastos ${ }^{7}$. Os procedimentos endodônticos, indicados em muitos destes casos, exigem um maior número de sessões clínicas e, provavelmente, têm custo mais elevado do que as restaurações.

O número de extrações teve menor redução quando comparado às restaurações. De forma semelhante aos procedimentos endodônticos, o número acentuado de extrações pode ser um indicador de agravamento das lesões cariosas. Apesar de ser um procedimento indicado para casos de lesões extensas em que os procedimentos endodôntico e/ou restaurador não são possíveis $^{28}$, deve-se considerar que a perda precoce de dentes decíduos é um fator de risco para maloclusões ${ }^{29,30}$.

Durante a pandemia, as crianças não puderam frequentar creches/escolas e aconselhou-se que permanecessem em casa. Até $60 \%$ dos traumatismos em dentes decíduos acontecem enquanto as crianças estão em casa e podem resultar de violência doméstica ou de quedas de bicicleta, patinete ou enquanto brincam ${ }^{31-33}$. Diante disso, é possível que tenha havido aumento na prevalência de traumatismo e, consequentemente, maior necessidade de extração. Os episódios de traumatismo podem justificar a pequena redução nos procedimentos endodônticos.

Houve uma correlação negativa entre o número de procedimentos odontológicos e de casos confirmados de COVID-19. 
Ao longo do período investigado, o aumento do número de casos de contaminação pelo coronavírus exigiu alterações em medidas sanitárias e restrição de atendimentos odontológicos. O menor número de procedimentos pode se relacionar ainda à recusa do cuidador em levar a criança à consulta devido ao receio de contrair a infecção. De acordo com estudos prévios, os pacientes adultos têm receio de ir ao consultório odontológico e sugerem que há grande risco de contrair a COVID-19 neste ambiente ${ }^{34,35}$.

É inquestionável que as medidas adotadas para minimizar a infecção pelo coronavírus são necessárias e importantes para conter o avanço da COVID-19. Entretanto, ao se considerar que a cárie dentária tem elevada prevalência e causa impacto negativo na qualidade de vida e no desenvolvimento infantil, deve-se estar ciente que estratégias para lidar com esta doença e, na medida do possível, oferecer tratamento às crianças devem ser repensadas no cenário da pandemia. Publicações nacionais e internacionais propõem estratégias para o atendimento odontopediátrico ${ }^{36-38}$. A recomendação de adotar procedimentos minimamente invasivos para o manejo das lesões de cárie ganhou destaque ${ }^{39}$. Procedimentos como a aplicação de diamino fluoreto de prata e o tratamento restaurador atraumático, dentre outros, são reconhecidos por gerar menor quantidade de aerossol, além de preservar estrutura dentária, reduzir o risco de acometimento do tecido pulpar e a necessidade de terapias mais invasi$\operatorname{vas}^{39,40}$. Estes procedimentos são opções para evitar o adiamento do cuidado às crianças com cárie tanto no cenário da pandemia quanto no pós-pandemia. No contexto do traumatismo dentário, outra condição bucal com elevada frequência em crianças, cuidadores e crianças devem ser orientados sobre medidas preventivas como uso de capacete ao andar de bicicleta $^{33}$ e a supervisão da criança.

Neste estudo foram analisados apenas os dados sobre procedimentos odontológicos realizados em dentes decíduos de crianças atendidas em Goiás. Isso impede a generalização dos 
resultados para crianças em dentição mista e permanente, assim como para aquelas atendidas em outros estados. Por outro lado, esta análise permite reconhecer as necessidades das crianças goianas e elaborar medidas específicas para esta população. Medidas como a capacitação dos profissionais para usarem os procedimentos minimamente invasivos e a educação em saúde bucal das crianças e das suas famílias são desejáveis.

\section{Conclusão}

Concluiu-se que a pandemia de COVID-19 impactou negativamente no número de procedimentos odontopediátricos realizados nos serviços públicos de Goiás. A redução do número de procedimentos pode ser encarada como um indicador para o agravamento da condição bucal infantil e a maior necessidade de procedimentos. Os profissionais e os gestores dos serviços de saúde devem elaborar estratégias para atender ao possível aumento de demanda de procedimentos e lidar com as consequências dos problemas bucais na qualidade de vida das crianças.

\section{Referências}

1 - Uribe SE, Innes N, Maldupa I. The global prevalence of early childhood caries: a systematic review with meta-analysis using the WHO diagnostic criteria. Int J Paediatr Dent. 2021; 31(6): 817-830. Doi: 10.1111/ipd.12783.

2 - Kassebaum NJ, Bernabé E, Dahiya M, Bhandari B, Murray CJL, Marcenes W. Global burden of untreated caries: a systematic review and metaregression. J Dent Res. 2015; 94(5): 650-658.

3 - AAPD. Policy on Early Childhood Caries (ECC): classifications, consequences, and preventive strategies. Pediatr Dent. 2017; 39(7 Suppl): 59-61.

4 - Corrêa-Faria P, Daher A, Freire MCM, Abreu MHN, Bönecker M, Costa LR. Impact of untreated dental caries severity on the quality of life of preschool children and their families: a cross-sectional study. Qual Life Res. 2018; 27(12): 3191-3198.

5 - Patnana AK, Chugh A, Chugh VK, Kumar P, Vanga NRV, Singh S. The prevalence of traumatic dental injuries in primary teeth: a systematic review and meta-analysis. Dent Traumatol. 2021; 37(3): 383-399. 
6 - Lombardo G, Vena F, Negri P, Pagano S, Barilotti C, Paglia L et al. Worldwide prevalence of malocclusion in the different stages of dentition: a systematic review and meta-analysis. Eur J Paediatr Dent. 2020; 21(2): 115-122.

7 - Casamassimo PS, Thikkurissy S, Edelstein BL, Maiorini E. Beyond the dmft: the human and economic cost of early childhood caries. J Am Dent Assoc. 2009; 140(6): 650-657.

8 - Feldens CA, Kramer PF, Feldens EG, Pacheco LM, Vítolo MR. Socioeconomic, behavioral, and anthropometric risk factors for traumatic dental injuries in childhood: a cohort study. Int J Paediatr Dent. 2014; 24(3): 234-243.

9 - BaniHani A, Deery C, Toumba J, Munyombwe T, Duggal M. The impact of dental caries and its treatment by conventional or biological approaches on the oral health-related quality of life of children and carers. Int J Paediatr Dent. 2018; 28(2): 266-276.

10 - Innes NPT, Chu CH, Fontana M, Lo ECM, Thomson WM, Uribe S et al. A century of change towards prevention and minimal intervention in cariology. J Dent Res. 2019; 98(6): 611-617.

11 - Pucca Jr. GA, Gabriel M, Araujo ME, Almeida FC. Ten Years of a National Oral Health Policy in Brazil: Innovation, Boldness, and Numerous Challenges. J Dent Res. 2015; 94(10): 1333-1337.

12 - Feldens CA, Fortuna MJ, Kramer PF, Ardenghi TM, Vítolo MR, Chaffee BW. Family Health Strategy associated with increased dental visitation among preschool children in Brazil. Int J Paediatr Dent. 2018; 28(6): 624-632.

13 - Camargo MBJ, Barros AJD, Frazão P, Matijasevich A, Santos IS, Peres MA et al. Predictors of dental visits for routine check-ups and for the resolution of problems among preschool children. Rev Saude Publica. 2012; 46(1): 87-97.

14 - Goettems ML, Ardenghi TM, Demarco FF, Romano AR, Torriani DD. Children's use of dental services: influence of maternal dental anxiety, attendance pattern, and perception of children's quality of life. Community Dent Oral Epidemiol. 2012; 40(5): 451-458.

15 - Curi DSC, Figueiredo ACL, Jamelli SR. Fatores associados à utilização dos serviços de saúde bucal pela população pediátrica: uma revisão integrativa. Cienc Saude Coletiva. 2018; 23(5): 1561-1576.

16 - Palacios Cruz M, Santos E, Velázquez Cervantes MA, León Juárez M. COVID-19, a worldwide public health emergency. Rev Clin Esp (Barc). 2021; 221(1): 55-61.

17 - Epstein JB, Chow K, Mathias R. Dental procedure aerosols and COVID-19. Lancet Infect Dis. 2021; 21(4): e73. 
18 - Moraes RR, Correa MB, Queiroz AB, Daneris Â, Lopes JP, PereiraCenci $T$ et al. COVID-19 challenges to dentistry in the new pandemic epicenter, Brazil. PLoS One. 2020; 15(11): e0242251. Doi: 10.1371/ journal.pone.0242251.

19 - Chisini LA, Costa FS, Demarco GT, Silveira ER, Demarco FF. COVID-19 pandemic impact on paediatric dentistry treatments in the Brazilian Public Health System. Int J Paediatr Dent. 2021; 31(1): 31-34.

20 - Brasil. Secretaria de Estado de Goiás. Governo do Estado de Goiás. Boletins e Informes [Internet]. Goiânia: Secretaria de Estado de Goiás, 2021. [citado em 20 maio 2021]. Disponível em: https://www.saude. go.gov.br/boletins-e-informes

21 - Brasil. Ministério da Saúde. Nota técnica nº9. Covid -19 e atendimento odontológico no SUS [internet]. Brasília, DF: Ministério da Saúde, 2021. [citado em 20 maio 2021]. Disponível em: https://kidopilabs.com.br/ planificasus/upload/covid19_anexo_11.pdf

22 - American Dental Association. Summary of ADA Guidance During the SARS-CoV-2 Crisis. [internet]. [citado em 18 maio 2021]. Disponível em: https:/www.wsda.org/docs/librariesprovider52/default-documentlibrary/summary-of-ada-guidance-during-the-sars-cov-2-crisis. pdf?sfvrsn=0.

23 - American Dental Association (ADA). What Constitutes a Dental Emergency? [internet]. Kansas: ADA.org, 2020. [citado em 20 maio 2021]. Disponível em: https://success.ada.org/ /media/CPS/Files/ Open\%20Files/ADA_COVID19_Dental_Emergency_DDS.pdf

24 - Conselho Regional de Odontologia de Goiás (CRO-GO). Recomendações da câmara técnica de odontologia para pacientes com necessidades especiais do estado de Goiás frente ao atendimento em tempos de COVID-19. [internet]. Goiânia: CRO-GO, 2020. [citado em 20 maio 2021]. Disponível em: http://www.crogo.org.br/site/index. php/destaques/777-nota-de-recomendacoes-da-camara-tecnica-deodontologia-para-pacientes-com-necessidades-especiais-do-estadode-goias-frente-ao-atendimento-em-tempos-de-covid-19

25 - Brasil. Ministério da Saúde. Agência Nacional de Vigilância Sanitária. Nota Técnica GVIMS/GGTES/ANVISA № 04/2020, de 31 de março de 2020. [internet]. Brasília, DF: Ministério da Saúde, 2020. [citado em 20 maio 2021]. Disponível em: http://portal.anvisa.gov.br/documents/33852/271858/ Nota+T\%C3\%A9cnica+n+04-2020+GVIMS-GGTES-ANVISA/ab5986603de4-4f14-8e6f-b9341c196b28

26 - Pitts NB, Baez RJ, Diaz-Guillory C, Donly KJ, Feldens CA, McGrath $\mathrm{C}$ et al. Early Childhood Caries: IAPD Bangkok Declaration. J Dent Child. 2019; 86(2): 72. 
27 - Chaurasiya A, Gojanur S. Evaluation of the clinical efficacy of 38\% silver diamine fluoride in arresting dental caries in primary teeth and its parental acceptance. J Indian Soc Pedod Prev Dent. 2021; 39(1): 85-89.

28 - Coll JA, Dhar V, Vargas K, et al. Use of non-vital pulp therapies in primary teeth. Pediatr Dent 2020; 42(5): 337-349.

29 - Bhujel N, Duggal MS, Saini P, Day PF. The effect of premature extraction of primary teeth on the subsequent need for orthodontic treatment. Eur Arch Paediatr Dent. 2016; 17(6): 423-434.

30 - Van der Weijden FN, Hesse D, Americano GCA, Sociero VM, Bonifacio CC. The effect of pulp inflammation and premature extraction of primary molars on the successor permanent teeth. A retrospective study. Int J Paediatr Dent. 2020; 30(1):18-26.

31 - Coulthard P, Hutchison I, Bell JA, Coulthard ID, Kennedy H. COVID19 , domestic violence and abuse, and urgent dental and oral and maxillofacial surgery care. Br Dent J. 2020; 228(12): 923-926.

32 - Bastone EB, Freer TJ, McNamara JR. Epidemiology of dental trauma: a review of the literature. Aust Dent J. 2000; 45(1): 2-9.

33 - Ilyas N, Green A, Karia R, Sood S, Fan K. Demographics and management of paediatric dental-facial trauma in the 'lockdown' period: a UK perspective. Dent Traumatol. 2021; 37(4): 576-582.

34 - Moffat RC, Yentes CT, Crookston BT, West JH. Patient perceptions about professional dental services during the COVID-19 pandemic. JDR Clin Trans Res 2021; 6(1): 15-23.

35 - Martina S, Amato A, Faccioni P, Iandolo A, Amato M, Rongo R. The perception of COVID-19 among Italian dental patients: an orthodontic point of view. Prog Orthod. 2021; 22(1):11.

36 - Ilyas N, Agel M, Mitchell J, Sood S. COVID-19 pandemic: the first wave: an audit and guidance for paediatric dentistry. Br Dent J. 2020; 228(12): 927-931.

37 - Amorim LM, Maske TT, Ferreira SH, Santos RB, Feldens CA, Kramer PF. New post-COVID-19 biosafety protocols in pediatric dentistry. Pesqui Bras Odontopediatria Clín Integr. 2020; 20(supp1): e0117.

38 - Barbosa MG, Silva NR, Oliveira SSB, Corrêa-Faria P. Manejo da cárie dentária e comportamento infantil durante a pandemia de COVID-19: relato de caso. Rev Odontol Bras Central. 2021; 30(89): 209-221.

39 - Asociación Latinoamericana de Odontopediatría. Tratamiento de caries en época de COVID-19: Protocolos clínicos para el control de generación de aerosoles. Rev Latinoam Odontop. 2020; 10(2): 1-28.

40 - Innes NP, Manton DJ. Minimum intervention children's dentistry: the starting point for a lifetime of oral health. Br Dent J. 2017; 223(3): 205-213. 


\title{
How has the Covid-19 pandemic affected pediatric dental treatment in public health services? Data analysis of the State of Goiás
}

\begin{abstract}
Objectives: To evaluate the impact of the COVID-19 pandemic on the dental treatment of children attended in the public service in Goiás and the relationship between the number of cases of the disease and pediatric dental procedures. Material and method: Data on the number of dental procedures and confirmed cases of COVID-19 were obtained in consultation with the public reports made available by the Ministry of Health and epidemiological bulletins of health agencies. The difference in the number of procedures was calculated: 1) comparison of the number of procedures performed in February and March 2020, when the first case in Goiás was confirmed; 2) and the number of procedures performed in each month of 2020 with its correspondent in 2019. The results were obtained in absolute number and in percentage of increase/decrease. The correlation between the number of procedures and cases of COVID-19 was evaluated. Results: In the first month of the pandemic, there was a $24 \%$ reduction in total procedures. Greater reduction was observed for restorations (28\%), followed by extractions (21\%). When comparing the months of 2020 with the respective months of 2019, there was a reduction of up to $83 \%$ in the total procedures; in the second half of the year, the reduction ranged from $76 \%$ to $83 \%$. There was a reduction of up to $100 \%$ in the number of restorations. A smaller reduction was observed in the number of endodontic procedures (23 to $88 \%$ ). There was a negative correlation between the number of procedures and cases of COVID-19 (rho $-0.806 ; \mathrm{p}=0.005)$. The pandemic negatively impacted the number of pediatric dental procedures performed in the SUS in Goiás. A greater number of cases of COVID-19 was related to the smaller number of pediatric dental procedures.
\end{abstract}

KEYWORDS: Coronavirus Infections; Dental care; Unified Health System.

\section{Como citar este artigo}

Florêncio MMC, Vaz GG, Araújo RLF, Corrêa-Faria P. Como a pandemia de Covid-19 afetou o tratamento odontopediátrico nos serviços públicos de saúde? Análise de dados do Estado de Goiás. Rev Odontol Bras Central 2021; 30(89): 434-447. DOI: 10.36065/robrac.v30i89.1542 